\title{
Commentary on "Temporal Dynamics of Cerebral Blood Flow During the Acute Course of Severe Subarachnoid Hemorrhage Studied by Bedside Xenon-Enhanced CT"
}

\author{
Rajat Dhar ${ }^{*}$ (D)
}

@ 2019 Springer Science+Business Media, LLC, part of Springer Nature and Neurocritical Care Society

The rupture of an intracranial aneurysm into the subarachnoid space triggers a complex cascade of cerebral and systemic events that places the patient at risk of early and delayed cerebral ischemia (DCI). Cerebral infarcts and DCI are more strongly associated with poor outcomes after subarachnoid hemorrhage (SAH) than intermediate processes such as cerebral vasospasm [1]. Yet our understanding of the origins and correlates of ischemia and infarction after SAH remains incomplete. The paradigm that emerged from early physiologic studies in $\mathrm{SAH}$ patients was that while cerebral blood flow (CBF) may be impaired early after $\mathrm{SAH}$, this is coupled with a reduction in cerebral metabolism and likely does not represent primary ischemia in most patients [2, 3]. However, those that develop arterial narrowing from vasospasm and delayed neurological deterioration have further reductions in CBF that are associated with elevated oxygen extraction and place them at risk of cerebral infarction. Core aspects of this paradigm have been challenged in the past decade as studies have both shown that vasospasm is not always associated with reductions in CBF and that improvements in vasospasm are not reliably coupled with reductions in infarction or improved clinical outcomes $[4,5]$. Nevertheless, cerebral perfusion and CBF remain a critical aspect of the physiologic management

\footnotetext{
*Correspondence: dharr@wustl.edu

Department of Neurology (Division of Neurocritical Care), Washington University in Saint Louis, 660 S Euclid Avenue, Campus Box 8111, Saint Louis, MO 63110, USA
}

This comment refers to the article available at https://doi.org/10.1007/ s12028-019-00675-x. of SAH patients; for example, permissive and induced hypertension are routinely employed to prevent and treat those with DCI [6]. However, even in this respect, recent studies have challenged the central tenet that raising blood pressure reliably augments CBF [7]. Conversely, we now recognize that aggressive hemodynamic augmentation poses risks including hypertensive encephalopathy (also known as posterior reversible encephalopathy syndrome or PRES) that may reflect the consequences of excessive cerebral perfusion pressures [8].

Therefore, an evaluation of the dynamics of CBF after SAH may provide us a sense of how to better understand the relationship of CBF to DCI and outcomes. It is such a study that has been performed by Enquist et al. They employed bedside xenon-enhanced computed tomography $(\mathrm{CT})$ to measure cortical blood flow in $51 \mathrm{SAH}$ patients at both early (day $0-3$ ) and subacute (day 4-7) time points. Importantly, this study was restricted to only those remaining intubated at both time points and so is skewed toward only the most severe SAH patients who did not improve adequately to be extubated (30 of the 81 initially high-grade SAH patients with baseline studies could not be included due to subsequent improvement). It could also not include those who had good early clinical grade but subsequently deteriorated from DCI. In the select subgroup with studies at both time points, they found that median cortical $\mathrm{CBF}$ at day $0-3$ was $32.8 \mathrm{ml} / 100 \mathrm{~g} / \mathrm{min}$ with $17 \%$ of regions studied having CBF below $20 \mathrm{ml} / 100 \mathrm{~g} / \mathrm{min}$. They did not find any significant change in CBF or proportion of regions with low flow on repeat studies at days 4-7 in the overall cohort. However, when they divided subjects into those with 
low baseline CBF (somewhat arbitrarily defined as below $30 \mathrm{ml} / 100 \mathrm{~g} / \mathrm{min}$ ) versus normal CBF, they found that those with low CBF demonstrated improvements over time while the remainder did not.

A significant complicating factor in interpreting these findings was whether $\mathrm{HHH}$-therapy (hypertension, hypervolemia, and hemodilution) was provided (as it was to 22 of the 51 subjects). This therapy was used in cases of neurological deterioration and included fairly mild degrees of induced hypertension (systolic blood pressure above $140 \mathrm{~mm} \mathrm{Hg}$ ) as well as infusions of dextran and albumin (targeting central venous pressure of 8-12 $\mathrm{mm}$ $\mathrm{Hg})$. Those who did not receive HHH-therapy showed no improvement in CBF over time (i.e., those with low CBF remained low) while those receiving $\mathrm{HHH}$-therapy appeared to exhibit a marked increase in CBF (from 21.3 to $37.8 \mathrm{ml} / 100 \mathrm{~g} / \mathrm{min}$ ).

There are a number of caveats when drawing conclusions from these perplexing but provocative physiologic results. Firstly, the two CBF measurements were separated by several days during which time multiple interventions and alterations in cerebral and systemic physiology likely occurred. They also lie in contrast to the recently reported results of the (admittedly small) HIMALAIA (Hypertension Induction in the Management of AneurysmaL subArachnoid hemorhage with secondary IschemiA) study group which used CT perfusion to measure CBF at time of DCI onset compared with 24-36 h after hypertensive therapy and found no significant rise in blood flow compared to a comparable control group not receiving induced hypertension (mean arterial pressure [MAP] was on average $12 \mathrm{~mm} \mathrm{Hg}$ higher with the intervention) [7]. These findings mirror those of our own group, who used positron emission tomography to measure CBF before and immediately after raising MAP by an average of $25 \mathrm{~mm} \mathrm{Hg}$ but still found no overall or selective rise in CBF (even in low flow regions) [9]. In light of these studies, it is difficult to reconcile and interpret the findings of the present study. The authors conclude (with appropriate caution) that $\mathrm{HHH}$-therapy could have an influence on CBF, especially in those with low baseline CBF. Conversely, they also postulate that those with low CBF who did not receive HHH-therapy may have had covert ischemia which was not detected or treated. It seems that those with DCI (who were treated) had better CBF than those without or perhaps not recognized to have DCI (although outcomes, as studied, were not different between groups). The authors argue that poor-grade SAH patients may benefit from evaluation of $\mathrm{CBF}$ and greater surveillance for ischemia in those with impaired baseline flow. This approach has merits but requires more focused study given the significant selection bias and potentially confounded nature of their findings (i.e., effect of $\mathrm{HHH}$-therapy overlapping entirely with effect of DCI so we cannot tell what is really primary or causative). While we cannot draw definitive conclusions from their results and should not change our practice at this point, the authors should be congratulated for dissecting the dynamic physiology of blood flow after SAH and pointing out the unresolved questions that remain in this arena.

\section{Publisher's Note}

Springer Nature remains neutral with regard to jurisdictional claims in published maps and institutional affiliations.

Published online: 27 February 2019

\section{References}

1. Vergouwen MDI, et al. Lower incidence of cerebral infarction correlates with improved functional outcome after aneurysmal subarachnoid hemorrhage. J Cereb Blood Flow Metab. 2011;31:1545-53.

2. Carpenter DA, et al. Cerebral oxygen metabolism after aneurysmal subarachnoid hemorrhage. J Cereb Blood Flow Metab. 1991;11:837-44.

3. Kawamura S, et al. Sequential changes in cerebral blood flow and metabolism in patients with subarachnoid haemorrhage. Acta Neurochir (Wien). 1992;114(1-2):12-5.

4. Dhar R, et al. Relationship between angiographic vasospasm and regional hypoperfusion in aneurysmal subarachnoid hemorrhage. Stroke. 2012;43:1788-94.

5. Etminan $\mathrm{N}$, et al. Effect of pharmaceutical treatment on vasospasm, delayed cerebral ischemia, and clinical outcome in patients with aneurysmal subarachnoid hemorrhage: a systematic review and meta-analysis. J Cereb Blood Flow Metab. 2011;31:1443-51.

6. Kassell NF, et al. Treatment of ischemic deficits from vasospasm with intravascular volume expansion and induced arterial hypertension. Neurosurgery. 1982;11:337-43.

7. Gathier CS, et al. Effects of induced hypertension on cerebral perfusion in delayed cerebral ischemia after aneurysmal subarachnoid hemorrhage: a randomized clinical trial. Stroke. 2015;46(11):3277-81.

8. Allen ML, et al. Posterior reversible encephalopathy syndrome as a complication of induced hypertension in subarachnoid hemorrhage: a case-control study. Neurosurgery. 2018. https://doi.org/10.1093/neuros/ nyy240.

9. Diringer MN, et al. Effect of high-dose simvastatin on cerebral blood flow and static autoregulation in subarachnoid hemorrhage. Neurocrit Care. 2016;25(1):56-63. 